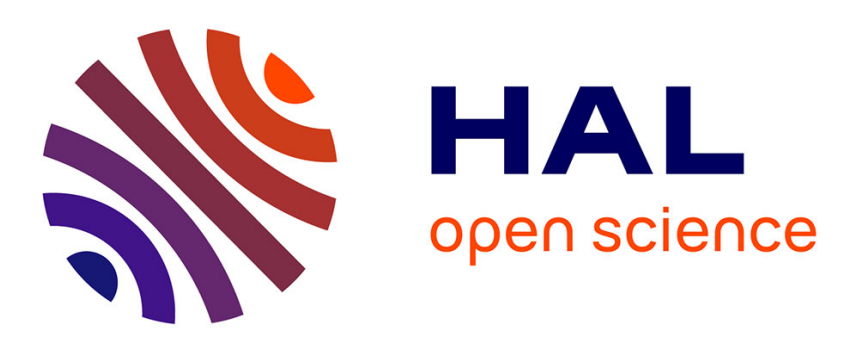

\title{
ANALYTICAL DETERMINATION OF INITIAL CONDITIONS LEADING TO FIRING IN NERVE FIBERS
}

\author{
Sabir Jacquir, Stéphane Binczak, Jean-Marie Bilbault
}

\section{- To cite this version:}

Sabir Jacquir, Stéphane Binczak, Jean-Marie Bilbault. ANALYTICAL DETERMINATION OF INITIAL CONDITIONS LEADING TO FIRING IN NERVE FIBERS. International journal of bifurcation and chaos in applied sciences and engineering , 2007, 17 (10), pp.3697-3701. hal-00584230

\section{HAL Id: hal-00584230 \\ https://u-bourgogne.hal.science/hal-00584230}

Submitted on 11 Apr 2011

HAL is a multi-disciplinary open access archive for the deposit and dissemination of scientific research documents, whether they are published or not. The documents may come from teaching and research institutions in France or abroad, or from public or private research centers.
L'archive ouverte pluridisciplinaire HAL, est destinée au dépôt et à la diffusion de documents scientifiques de niveau recherche, publiés ou non, émanant des établissements d'enseignement et de recherche français ou étrangers, des laboratoires publics ou privés. 


\title{
ANALYTICAL DETERMINATION OF INITIAL CONDITIONS LEADING TO FIRING IN NERVE FIBERS
}

\author{
SABIR JACQUIR, STEPHANE BINCZAK, JEAN-MARIE BILBAULT \\ Laboratoire LE2I, UMR CNRS 5158, Université de Bourgogne, 9 avenue Alain Savary \\ Dijon cedex, 21078, France \\ sjacquir@u-bourgogne.fr, stbinc@u-bourgogne.fr \\ http://<www.le2i.com $>$ \\ Received (Day Month Year) \\ Revised (Day Month Year)
}

\begin{abstract}
An analytical solution characterizing initial conditions leading to action potential firing in smooth nerve fibers is determined, using the bistable equation. In the first place, we present a non-trivial stationary solution wave, then, using the perturbative method, we analyze the stability of this stationary wave. We show that it corresponds to a frontier between the initiation of the travelling waves and a decay to the resting state. Eventually, this analytical approach is extended to FitzHugh-Nagumo model.

Keywords: Stationarity; reaction-diffusion; nerve fibers.
\end{abstract}

\section{Introduction}

In 1952, Hodgkin and Huxley (HH) [Hodgkin and Huxley (1952)] described the behaviour of electrical impulses in the axon of the squid by a set of partial differential equations. Their model produces a realistic description of the action potential propagation along a smooth axon. There are other models which arise from the simplification of the HH model, such as the FitzHugh-Nagumo (FHN) model [FitzHugh (1961); Nagumo et al. (1962)]. This one produces the qualitative dynamics of the nerve membrane and it is largely used to investigate the mechanism of the propagation or its failure in a one-dimensional excitable medium [Neu et al. (1997); Lewis and Keener (2000)], in inhomogeneous media [Prat and Li (2003)], and in discrete reaction-diffusion systems [Binczak and Bilbault (2004); Keener (1991); Bell (1990)], for instance. In a piecewise form, it has also been used to study threshold phenomena for the reaction-diffusion systems [Terman (1983)]. When focussing on the leading edge of impulses, this FHN model reduces to the bistable equation. The aim of this paper is to determine the conditions allowing the excitation of nerve fibers (initiation of the travelling pulse) or its setting in latency. The paper is organized as follows: In section 2 , the analytical shape of the stationary wave which corresponds to initial conditions, is calculated and its stability is studied for the bistable equation. The generalization of the stationary solution to FHN model is presented in section 3. Finally, the section 4 is devoted to concluding remarks. 


\section{Stationarity in the Bistable Model}

In a first place, let us consider the bistable equation in an infinite continuous onedimensional medium such as:

$$
\frac{\partial V}{\partial t}=D \frac{\partial^{2} V}{\partial x^{2}}-f(V)
$$

In this model of the smooth nerve fiber, $V$ denotes the voltage accross the membrane, $D$ represents the diffusion coefficient, $t$ is the time and $x$ is the longitudinal axis of the fiber. The nonlinear function $f(V)=V(V-\alpha)(V-1)$ represents the behaviour of the sodium current with $\alpha$ acting as a threshold between the passive and the active role of the sodium conductance $(0<\alpha<1)$, so that the steady state $V=0$ is the resting state and the steady state $V=1$ is the excited one. The system is completed by Neumann conditions, so that $\lim _{x \rightarrow \pm \infty} \frac{d V}{d x}=0$. In this context, we look for non uniform stationary waves so that $\lim _{x \rightarrow \pm \infty} V(x)=V_{\infty}$ and $V(x=0) \neq V_{\infty}$, where $V_{\infty}$ is the steady state 0 or 1 . Due to symmetry, we can reduce this problem to a semi-infinite medium by setting $\frac{d V}{d x}(x=0)=0$. Depending on the choice of $V_{\infty}$, several results are to be distinguished to find a stationary wave.

\subsection{Case where $V_{\infty}=0$}

We look for a time-independent solution $V=V_{s}(x)$ of eq. (1):

$$
D \frac{d^{2} V}{d x^{2}}=V(V-\alpha)(V-1) \text {. }
$$

Multiplying both sides by $\frac{d V}{d x}$ and taking into account the Neumann conditions requirements, eq. (2) yields, after integration, to

$$
\frac{d V}{d x}=\sqrt{\frac{2}{D}\left(\frac{V^{4}}{4}-(\alpha+1) \frac{V^{3}}{3}+\alpha \frac{V^{2}}{2}\right)}=\sqrt{\frac{2}{D} F(V)} .
$$

Where $F(V)$ is the antiderivative function of $f(V)$. In the case where $\alpha \leq 1 / 2, F(V)$ has three real roots and can be expressed by $F(V)=\frac{1}{4} V^{2}\left(V-V_{1}\right)\left(V-V_{2}\right)$ with $0<V_{1}<V_{2}$,

$$
V_{1,2}=\frac{2}{3}(\alpha+1) \mp \frac{1}{3} \sqrt{4 \alpha^{2}-10 \alpha+4},
$$

so that $V_{1} V_{2}=2 \alpha$. As illustrated in Fig. $1, V=0$ and $V=1$ are the minima of $F(V)$ corresponding to stable states and $V=\alpha$ is a local maximum corresponding to an unstable state. To integrate eq. (3), $F(V)$ must be positive, therefore excursion of $V$ must lie in the range $\left[0, V_{1}\right]$. Thus, eq. (3) leads to

$$
\int_{V_{1}}^{V_{s}} \frac{d V}{V \sqrt{\left(V-V_{1}\right)\left(V-V_{2}\right)}}= \pm \sqrt{\frac{1}{2 D}} \int_{0}^{x} d x .
$$

which yields to

$$
V_{s}(x)=\frac{V_{2}}{1+\left(\frac{V_{2}}{V_{1}}-1\right) \cosh ^{2}\left(\frac{x}{2} \sqrt{\frac{\alpha}{D}}\right)}
$$




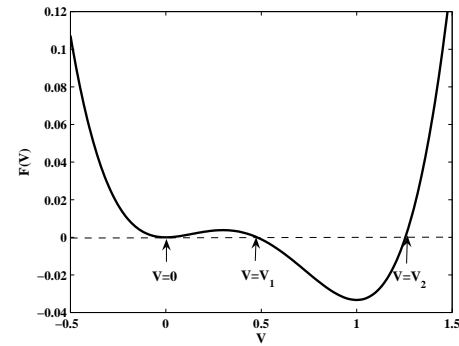

Fig. 1. F(V) in the case where $\alpha=0.3$.

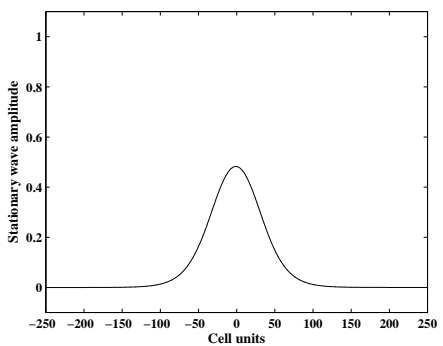

Fig. 2. Stationary wave in the case where $\alpha=0.3$

Eq. (6) corresponds to the stationary wave whose width depends on the diffusion parameter $D$ and on the threshold parameter $\alpha$. The amplitude of $V_{s}$ is maximum when $x=0$ so that $V_{s}(0)=V_{1}$ and is only a function of $\alpha$. Because of symmetry, this result can be extended to an infinite medium, as illustrated in Fig. 2. When $\alpha \ll 1$, eq. (6) becomes

$$
V_{s}(x)=\frac{3}{2} \alpha \operatorname{sech}^{2}\left(\frac{x}{2} \sqrt{\frac{\alpha}{D}}\right),
$$

which corresponds, when $D=1$, to the results obtained in [Neu et al. (1997)]. The existence of the stationary wave given by Eq. (6) (in accordance with the results obtained in [Bountis (2000)]) is assured only when $\alpha \in[0,1 / 2]$. As we shall see, the condition $\alpha \in[1 / 2,1]$ implies another solution.

\subsection{Case where $V \infty=1$}

In this case, eq. (2) yields to

$$
\frac{d V}{d x}=\sqrt{\frac{2}{D} F(V)} .
$$

The antiderivative function $F(V)$ has three real zeros only if $\alpha \geq 1 / 2$. In this case, it can be expressed by $F(V)=\frac{1}{4}(V-1)^{2}\left(V-V_{1}\right)\left(V-V_{2}\right)$ where $V_{1}<V_{2}<1$,

$$
V_{1,2}=\frac{2 \alpha}{3}-\frac{1}{3} \mp \frac{1}{3} \sqrt{4 \alpha^{2}+2 \alpha-2}
$$

As previously, to integrate eq. (8), $F(V)$ must be positive, therefore excursion of $V$ must lie in the range $\left[V_{2}, 1\right]$, as illustrated in Fig. 3. Thus, eq. (8) leads to

$$
\int_{V_{2}}^{V_{s}} \frac{d V}{(V-1) \sqrt{\left(V-V_{1}\right)\left(V-V_{2}\right)}}= \pm \sqrt{\frac{1}{2 D}} \int_{0}^{x} d x,
$$

which yields to

$$
V_{s}(x)=\frac{V_{1} \sinh ^{2}\left(\frac{\Gamma x}{2}\right)-V_{2} \cosh ^{2}\left(\frac{\Gamma x}{2}\right)+(1-2 \alpha) / 3}{V_{1} \cosh ^{2}\left(\frac{\Gamma x}{2}\right)-V_{2} \sinh ^{2}\left(\frac{\Gamma x}{2}\right)-1},
$$




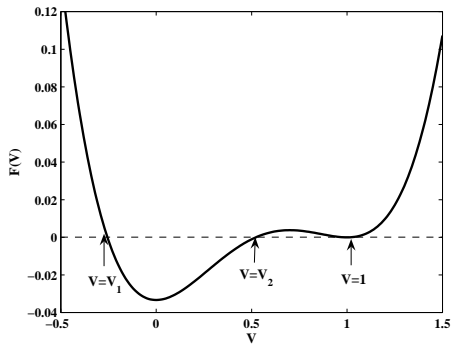

Fig. 3. F(V) in the case where $\alpha=0.7$.

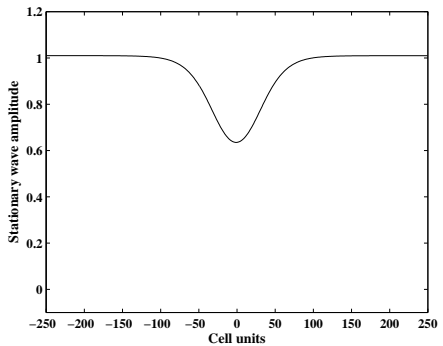

Fig. 4. Stationary wave in the case where $\alpha=0.7$.

with $\Gamma=\sqrt{D(1-\alpha)}$. Here again, the width of this stationary wave depends on $D$ and $\alpha$, while the minimum value of the amplitude $V_{\text {min }}=V_{2}$, located at $x=0$ is only function of $\alpha$. An illustration of such a wave is given in Fig. 4, where the medium is extended from a semi-infinite one to an infinite one. Note that the existence of this stationary wave is assured when $\alpha \in[1 / 2,1]$, complementing the first result.

\subsection{Stability of the stationary solution}

To study the stability of the stationary wave, we examine the evolution of an infinitesimal disturbance $v(x, t)$ superimposed on the stationary wave $V_{s}(x)$. To determine its evolution equation, we introduce a scaling parameter $\Psi \ll 1$ so that $V(x, t)$ can be expressed by

$$
V(x, t)=V_{s}(x)+\Psi v(x, t) .
$$

Equation (12) is inserted in eq. (1) leading to express the evolution of $V_{s}(x)$ and $v(x, t)$ separately. Using this perturbative method to the power of $\Psi$ inferior to 2, eq. (1) is developped into two equations depending on the power of $\Psi$ :

$$
\left\{\begin{array}{l}
\Psi^{0}: D \frac{d^{2} V s}{d x^{2}}=V s(V s-\alpha)(V s-1) \\
\Psi^{1}: \frac{\partial v}{\partial t}=D \frac{\partial^{2} v}{\partial x^{2}}-h(x) v,
\end{array}\right.
$$

with $h(x)=3 V_{s}(x)^{2}-2(\alpha+1) V_{s}(x)+\alpha$. The first equation $\left(\Psi^{0}\right)$ corresponds to the stationary case, while the second one $\left(\Psi^{1}\right)$ gives the evolution of the perturbation $v(x, t)$. Let us focus on a dirac perturbation located at $x=0$, as $v(x, t=0)=v_{0} \delta(x)$. In this case, one can approximate $h(x)$ so that $h(x)=h(0)=h_{0}$ becomes uniform in the region of $x=0$. Considering this approximation and using the Fourier transform and the Inverse Fourier transform, the solution of eq. (13) is given by:

$$
v(x, t)=v_{0} \frac{e^{-h_{0} t}}{\sqrt{4 \pi D t}} e^{-\frac{x^{2}}{4 D t}} .
$$

If $h_{0}>0$ then $v(x, t) \rightarrow 0$ therefore $V(x, t)=V_{s}(x, t)$, which indicates that the stationary wave is stable. If $h_{0}<0$ then $v(x, t)$ diverges, which is the case around $x=0 \forall \alpha$. This result indicates that this stationary wave is unstable and acts as a frontier between two 

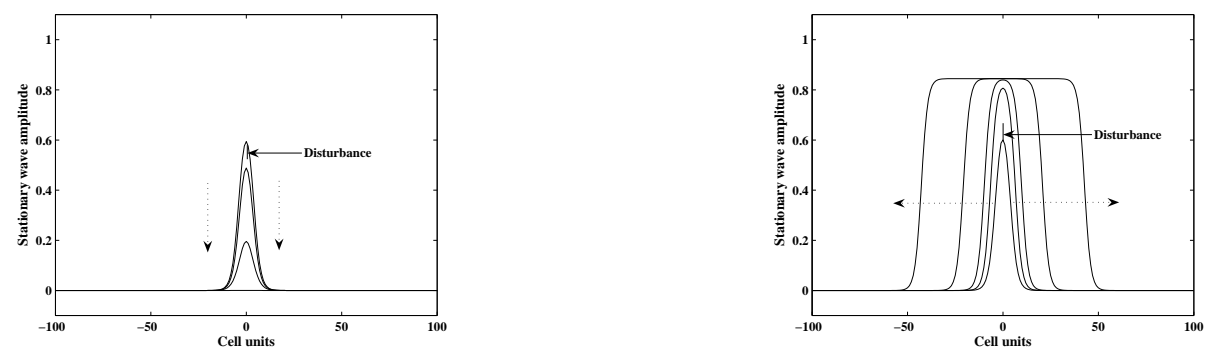

Fig. 5. Evolution of the perturbed stationary wave in the case where $\alpha=0.2$ and $D=1$.

different evolutions: From numerical simulations and according to [Neu et al. (1997)], if the initial signal is beneath $V_{s}(x)$, it will converge to the resting state $V(x, t)=0$, otherwise it generates a travelling wave, as illustrated in Fig 5, where, in the top (resp. bottom) part, a negative (resp. positive) perturbation is superimposed to the stationary solution, leading to a decay to zero (resp. a travelling wave). In the following section, the precedent results are generalized to FitzHugh-Nagumo model.

\section{Generalization to the FHN Model}

The FHN model includes a recovery variable variable $W$ indicating the capacity of the medium to revert to its resting state [Scott (1999)], and is expressed by

$$
\begin{aligned}
& \frac{\partial V}{\partial t}=D \frac{\partial^{2} V}{\partial x^{2}}-f(V)-W \\
& \frac{\partial W}{\partial t}=\varepsilon(V-\gamma W) .
\end{aligned}
$$

The stationary solution of this equation must satisfy

$$
D \frac{\partial^{2} V}{\partial x^{2}}-f(V)-\frac{V}{\gamma}=0 \text { and } W=V / \gamma
$$

Applying the previous methodology, the antiderivative function eq. (16) leads to

$$
\frac{d V}{d x}=\sqrt{\frac{2}{D} F(V)}
$$

where $F(V)=\frac{1}{4} V^{2}\left(V-V_{1}\right)\left(V-V_{2}\right)$,

$$
V_{1,2}=\frac{1}{3 \gamma}\left(2 \alpha \gamma+2 \gamma \mp \sqrt{4 \alpha^{2} \gamma^{2}-10 \alpha \gamma^{2}+4 \gamma^{2}-18 \gamma}\right)
$$

A nontrivial stationary wave only exists if $V_{1}$ and $V_{2}$ exist and are real, i.e. if $\alpha<\frac{1}{2}$ and $\gamma>\gamma_{c}>0$ with

$$
\gamma_{c}=\frac{9}{(1-2 \alpha)(2-\alpha)}>0
$$




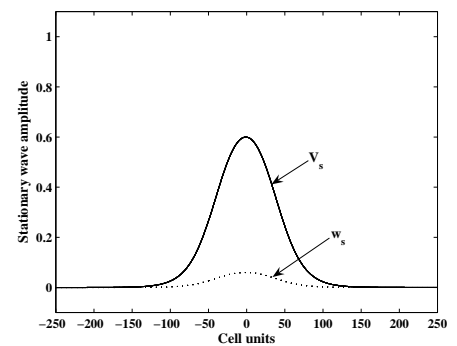

Fig. 6. Stationary wave of the FHN model $(\alpha=0.2$ and $\gamma=10)$.

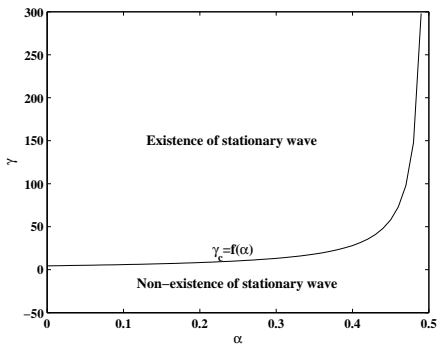

Fig. 7. $\gamma$ versus $\alpha$ implying the existence of stationary waves.

Under these conditions, integrating eq. (14) yields to express the stationary wave, so that

$$
\left\{\begin{array}{l}
V_{s}=\frac{V_{2}}{1+\left(\frac{V_{2}}{V_{1}}-1\right) \cosh ^{2}\left(\frac{x}{2} \sqrt{\frac{V_{1} V_{2}}{2 D}}\right)} \\
W_{s}=V_{s} \gamma^{-1}
\end{array}\right.
$$

An illustration of such a wave is presented in Fig. 6, while Fig. 7 shows the relationship between $\alpha$ and $\gamma$ leading to the existence of stationary waves, according to eq. (18). Numerical simulations have shown that a positive (resp. negative) perturbation superimposed to $V_{s}$ in the region of $x=0$ leads to the emergence of a travelling impulse (resp. a decay to zero), while a perturbation superimposed to $W_{s}$ implies an opposite evolution, which indicates that this stationary wave corresponds to minimum spatial initial conditions from which a travelling wave can emerge.

\section{Conclusion}

In this study, the criterion for the initiation of the travelling waves has been investigated, based on the existence of stationary waves. We suggest that these results could be applied to determine the minimum punctual electrode stimulation needed to generate an action potential. Referring to [Neu et al. (1997)], these stationary waves can be seen as some critical nucleus, which can be useful to determine the relationship between amplitude and width of conditions initiating travelling waves and to optimize the size of electrodes or stimulation areas.

\section{References}

Hodgkin, A.L. and Huxley, A.F. (1952). A quantitative description of membrane current and its application to conduction and excitation in nerve. J. Physiol., 117: 500-544.

FitzHugh, R. (1961). Impulses and physiological states in theoretical models of nerve membrane. Biophys. J., 1: 445-466.

Nagumo, J., Arimoto, S. and Yoshizawa, S. (1962). An active impulse transmission line simulating nerve axon. Proc. IRE., 50: 2061-2070.

Neu, J.C., Preissig, R.S. and Krassowska, W. (1997). Initiation of propagation in a one-dimensional excitable medium. Physica D, 102: 285-299. 
Lewis, T.J. and Keener, J.P. (2000). Wave-block in excitable media due to regions of depressed excitability. Siam J. Appl. Math., 61: 293-316.

Prat, A. and Li, Y.X. (2003). Stability of front solutions in inhomogeneous media. Physica D, 186: $50-68$.

Binczak, S. and Bilbault, J.M. (2004). Experimental propagation failure in a nonlinear elctrical lattice. Int. J. Bifurcation and chaos, 14: 1819-1830.

Keener, J.P. (1991). The effects of discrete gap junctions coupling on propagation in myocardium. $J$. Theor. Biol., 148: 49-82.

Bell, J. (1990). Excitability behavior of myelinated axon models, in reaction-diffusion equations. Eds Brown, K.J. and Lacey, A.A., 95-116.

Terman, D. (1983). Threshold Phenomena for a Reaction-Diffusion System. Journal of Differential Equations, 47: 406-443.

Scott, A.C. (1999). Nonlinear Science. Oxford University Press, NY.

Bountis, T., Starmer C.F. and Bezerianos A. (2000). Stationary Pulses and Waves Front Formation in an Excitable Medium. Progress of Theoretical Physics Supplement, 139: 12-33. 